\title{
DIETARY PATTERNS WERE ASSOCIATED WITH OBESITY PARAMETERS AMONG HEALTHY WOMEN
}

\author{
Ahmad Syauqy*, Etika Ratna Noer, Alifia Mukti Fajrani, Dewi Marfu'ah Kurniawati, Rachma Purwanti, \\ Ayu Rahadiyanti, Devi Elvina Rahma \\ Department of Nutrition Science, Faculty of Medicine, Universitas Diponegoro. J1. Prof. H. Soedarto, SH., Tembalang, Semarang City, Central Java \\ 50275, Indonesia \\ *Corresponding author: E-mail: syauqy@fk.undip.ac.id
}

\begin{abstract}
Background: Obesity is a growing major health problem in some developing countries including Indonesia. Study examined the association between dietary patterns and obesity parameters using both body mass index (BMI) and waist circumference (WC) among healthy women in Indonesia was still rare.

Objectives: The objective of this study was to evaluate the correlation between dietary patterns and obesity parameters using BMI and WC among healthy women.

Methods: This study used a cross-sectional design with consecutive sampling. Healthy women aged 20 and above were selected in this study. Dietary data were collected using a food frequency questionnaire (FFQ). For categorical data, chi-squared test was done to compare the differences in the characteristics of the subjects among tertiles of dietary patterns. For continuous variables, a general linear model test was used for comparison. To evaluate the association between dietary patterns and obesity parameters (BMI and WC), we used multiple linear regression analysis adjusting for multiple confounding variables (age, current smoking, current drinking, and physical activity).

Results: Dietary pattern 1 consisted of 12 food items: snack cooked with oil, fish and seafood, processed food, organ meats, meat, poultry, rice- or flour-based products, staples cooked with oil, sugary drinks, refined dessert, and tea and coffee. Dietary pattern 2 consisted of 5 food items: eggs, light-colored vegetables, dark-colored vegetables, fruits, and soybeans. Dietary pattern 3 consisted of 7 food items: milk products, legumes, processed fruits, wholegrain, snacks cooked without oil, root crops, and jam/honey. Dietary pattern 1 was positively associated with obesity parameters including BMI and WC $(P<0.05)$; whereas, dietary pattern 2 and dietary pattern 3 were inversely correlated $(P<0.05)$. Conclusions: Our study find that dietary patterns were associated with BMI and WC among healthy women.
\end{abstract}

Keywords: dietary patterns; obesity; body mass index; waist circumference; adults

\section{INTRODUCTION}

Obesity is a growing major health problem in some developing countries including Indonesia. ${ }^{1-3}$ Obesity may increase the risk of degenerative diseases such as dyslipidemia, hypertension, renal disorder, diabetes. Moreover, obesity has a significant effect on both morbidity and mortality among the Asian population. ${ }^{4}$ A previous study showed that the prevalence of obesity among Indonesian aged 19-55 years elevated from almost $20 \%$ in 2007 to $25 \%$ in $2010 .^{3}$

Some factors including lifestyle are associated with the etiology of obesity. ${ }^{5}$ Dietary patterns have shown a significant role in the rising prevalence of obesity. ${ }^{6}$ A previous study found that a dietary pattern characterized by sweets, protein, sodium, fat, and food additives has a positively associated with components of metabolic syndrome including central obesity among Taiwanese adults 7 . Meanwhile, a prudent dietary pattern characterized by dietary fiber, complex carbohydrate, antioxidants, vitamins, phytochemicals, minerals, unsaturated fat, prebiotics, and probiotics has a negatively correlated with central obesity. ${ }^{8}$
Previous studies have explored the association between dietary patterns and chronic diseases including obesity. ${ }^{7,9,10}$ Moreover, individuals who consumed more vegetables and fruits, as well as less animal protein and saturated fat, had a better quality of life. ${ }^{7,8}$ However, to the best of our knowledge, study examined the association between dietary patterns and obesity parameters using both body mass index (BMI) and waist circumference (WC) in Indonesia among healthy women was still rare. Moreover, BMI and WC were commonly used for obesity markers. Hence, the objective of this study was to evaluate the correlation between dietary patterns and obesity parameters using BMI and WC among healthy women in.

\section{METHODS}

\section{Study Design}

This study used a cross-sectional design with consecutive sampling in Semarang, Indonesia, in 2020. Healthy women aged 20 and above were selected in this study. Moreover, those who have chronic diseases such as liver disease, renal disorder, or cancer were excluded. In total, a number of 102 adults completed the examination. Ethical approval 
for this study was received from the research ethics committee of the Faculty of Medicine, Diponegoro University, Indonesia (Ethical Clearance No. 172/EC/KEPK/FK-UNDIP/VII/2020).

\section{Assessment of Dietary Intake.}

Dietary data were collected using a food frequency questionnaire (FFQ) administered by a trained dietary interviewer. The FFQ was contained 24 food items featuring typical Indonesian food patterns. ${ }^{11}$ We designed the FFQ to measure how frequently the participant consumed certain portions of each food item with an obvious definition of portion size in a month (i.e. total of servings per day or week from the lowest to the highest frequency). For instance, the questions for consumption of vegetables, the 5 answer selections: none or less than 1 bowl/week, 1-3 bowls/week, 4-6 bowls /week, 1 bowls /day, and 2 or more bowls /day. ${ }^{11,12}$

\section{Anthropometric Measurements}

The anthropometric measurements were assessed by a trained health professional using a standardized protocol. Height $(\mathrm{cm})$ and weight $(\mathrm{kg})$ were assessed by a bioelectrical impedance analysis tool (TANITA Health Equipment, Hongkong). Body mass index was counted as weight $(\mathrm{kg})$ divided by the square of height $\left(\mathrm{m}^{2}\right)$. Waist circumference measurement was done at the mid-point between the lowest rib and the iliac crest on standing position. ${ }^{13,14}$

\section{Covariates}

Demographic characteristics such as age and lifestyle including current smoking, drinking status, and physical activity were collected using an administered questionnaire. Current drinking and smoking status were categorized as no and yes. Physical activity was divided by low ( $<1 \mathrm{~h}$ a week), moderate (1-2 $\mathrm{h}$ a week), and high ( $>2 \mathrm{~h}$ a week). ${ }^{7}$

\section{Statistical Analysis}

We used principal component analysis (PCA) to find the dietary patterns. We utilized eigenvalues $>2$ to set dietary patterns. The factor loading of $\geq 0.30$ was applied in the grouping of dietary patterns. Factor scores were calculated for each pattern by counting up the intake of food items divided by factor loadings. The dietary patterns were further named based on the data analysis. Categorical variables and continuous variables are presented as a number or percentage and mean \pm standard deviation (SD), respectively. Chisquared test was done to analyze the relation between the categorical data (smoking, drinking, and physical activity) and dietary patterns. A general linear model test was used for comparing the continuous variables (BMI and WC) and dietary patterns. To evaluate the association between dietary patterns and anthropometric parameters (BMI and WC), we used multiple linear regression analysis adjusting for multiple confounding variables (age, current smoking, current drinking, and physical activity). All statistical analyses were performed by SPSS 23 (IBM Corp., Armonk, NY, USA).

Table 1. Groups of Food Items According to Nutrient Profiles

\begin{tabular}{ll}
\hline Food groups & Food items \\
\hline Milk products & Milk, cheese, yoghurt \\
Snack cooked with oil & Bakwan, batagor, risol, cakwe, martabak, pastel, cireng, pempek, fried banana, \\
& doughnut, fried fish ball, chips, cassava chips \\
Legumes & Pea, nut, bean, peanut coated with flour \\
Fish and seafood & Fish, squid, shrimp, crap \\
Processed food & Sausage, canned food, instant food, bakso \\
Eggs & Chicken eggs, duck eggs, quail eggs \\
Organ meats & Liver, kidney, heart \\
Meat & Beef, veal, lamb, goat, pork \\
Poultry & Chicken, duck, goose, pigeon \\
Light-colored vegetables & Cabbage, pechay, squash, radish \\
Dark-colored vegetables & Spinach, carrots, kale, buncis \\
Fruits & Banana, orange, pier, mango, papaya, avocado, watermelon \\
Processed fruits & Canned fruit, rujak, asinan \\
Rice- or flour-based products & Rice, noodle, plain bread \\
Wholegrain & Whole grains, whole wheat, mixed grains, brown rice, oatmeal \\
Snacks cooked without oil & Gethuk, serabi cake, putu cake \\
Staples cooked with oil & Fried rice, fried noodle, $k$ wetiau, uduk rice \\
Root crops & Boiled/steamed potato, taro, cassava \\
Root crops cooked with oil & Fried potato, taro, cassava \\
Soybeans & Steamed tempe, tahu, tofu \\
Jam/honey & Jam, honey \\
Sugary drinks & Soft drinks, soda, energy drinks, flavored fruit drinks, tea and coffee with sugar \\
Refined dessert & Butter bread, sweet bread, cake, cookies, bicuit \\
Tea and coffee & Tea, coffee \\
\hline
\end{tabular}




\section{RESULTS}

Table 1 describes the classification of the food items according to the nutrient profiles into 24 food groups: milk product, snack cook with oil, legumes, fish and seafood, processed food, eggs, organ meats, meat, poultry, light-colored vegetables, dark-colored vegetables, fruits, processed fruits, rice- or flourbased products, wholegrain, snacks cooked without oil, staples cook with oil, root crops, root crops cook with oil, soybeans, jam/honey, sugary drinks, refined dessert, and tea and coffee.

The dietary patterns analyzed by PCA are presented in Table 2 . These three patterns explained $38.32 \%$ of total variance. Dietary pattern 1 consisted of 12 food items: snack cook with oil, fish and seafood, processed food, organ meats, meat, poultry, rice- or flour-based products, staples cook with oil, sugary drinks, refined dessert, and fried soybeans. Dietary pattern 2 consisted of 5 food items: eggs, light-colored vegetables, dark-colored vegetables, fruits, and soybeans. Dietary pattern 3 consisted of 7 food items: milk products, legumes, processed fruits, wholegrain, snacks cooked without oil, root crops, and jam/honey.

Table 3-5 show the characteristics of participants across tertiles of each dietary pattern. In comparison with the subjects in the lowest tertile (T1) of dietary pattern 1, the subjects in the highest tertile (T3) tended to be older and have worse anthropometric parameters (all $p<0.05$ ) (Table 3). The subjects in the highest tertile (T3) of dietary pattern 2 tended to be younger and have better anthropometric parameters compared with those in the lowest tertile (all $p<0.05$ ) (Table 4). The subjects in the highest tertile of dietary pattern 3 were likely to be older and have better anthropometric parameters compared with those in the lowest tertile (all $p<0.05$ ) (Table $5)$.

Table 2. Factor Loadings of the Three Dietary Patterns Obtained from Principal Component Analysis.

\begin{tabular}{llll}
\hline & Dietary Pattern 1 & Dietary Pattern 2 & Dietary Pattern 3 \\
\hline $\begin{array}{l}\text { Milk products, } \\
\text { Snack cooked with oil }\end{array}$ & 0.346 & - & $\mathbf{0 . 4 8 1}$ \\
Legumes & $\mathbf{0 . 5 1 7}$ & 0.211 & \\
Fish and seafood & & & $\mathbf{0 . 4 5 4}$ \\
Processed food & $\mathbf{0 . 5 3 4}$ & -0.210 & 0.384 \\
Eggs & $\mathbf{0 . 5 3 6}$ & $\mathbf{0 . 5 3 6}$ & 0.315 \\
Organ meats & & & - \\
Meat & $\mathbf{0 . 4 9 4}$ & & - \\
Poultry & $\mathbf{0 . 3 6 9}$ & $\mathbf{0 . 7 3 4}$ & -0.212 \\
Light-colored vegetables & - & $\mathbf{0 . 8 0 2}$ & 0.245 \\
Dark-colored vegetables & - & $\mathbf{0 . 5 9 2}$ & 0.209 \\
Fruits & -0.206 & 0.239 & 0.374 \\
Processed fruits & & 0.375 & $\mathbf{0 . 4 5 0}$ \\
Rice or flour based products & $\mathbf{0 . 4 4 8}$ & & -0.286 \\
Wholegrain & & & $\mathbf{0 . 5 9 8}$ \\
Snacks cooked without oil & & & $\mathbf{0 . 6 7 2}$ \\
Staples cooked with oil & $\mathbf{0 . 5 9 0}$ & & $\mathbf{0 . 3 7 5}$ \\
Root crops & & & \\
Root crops cooked with oil & $\mathbf{0 . 5 9 0}$ & $\mathbf{0 . 7 7 0}$ & $\mathbf{0 . 4 0 4}$ \\
Soybeans & & & -0.407 \\
Jam/honey & $\mathbf{0 . 4 5 8}$ & & -0.560 \\
Sugary drinks & $\mathbf{0 . 6 2 3}$ & 0.397 & \\
Refined dessert & & \\
Tea and coffee & $\mathbf{0 . 3 9 8}$ & & \\
\hline
\end{tabular}

Factor loadings with absolute values $<0.2$ are not presented.

Table 3. Characteristics of Participants in Dietary Pattern $1(n=102)$

\begin{tabular}{lllll}
\hline & Dietary Pattern 1 & & T3 & $\boldsymbol{p}$ \\
\cline { 2 - 5 } & T1 & T2 & $35.77 \pm 6.08$ & 0.039 \\
\hline Age (years) & $32.36 \pm 5.82$ & $34.50 \pm 6.78$ & 13.3 & 0.607 \\
Current smoker (yes) & 12.9 & 21.7 & 3.2 & 0.458 \\
Current drinker (yes) & 3.2 & 0 & 35.5 & 0.680 \\
Physical activity (low) & 41.9 & 36.0 & $34.78 \pm 7.15$ & 0.001 \\
Body mass index $\left(\mathrm{kg} / \mathrm{m}^{2}\right)$ & $29.21 \pm 5.11$ & $29.81 \pm 6.28$ & $98.90 \pm 14.36$ & $<0.001$ \\
Waist circumference $(\mathrm{cm})$ & $87.25 \pm 11.10$ & $89.55 \pm 9.62$ & & \\
\hline
\end{tabular}

Value are served as \% for categorical variables or mean \pm SD for continuous variables 
Table 4. Characteristics of Participants in Dietary Pattern $2(n=102)$

\begin{tabular}{lllll}
\hline & Dietary Pattern 2 & & T3 & p \\
\cline { 2 - 5 } & T1 & T2 & $31.73 \pm 6.12$ & 0.028 \\
\hline Age (years) & $35.51 \pm 6.45$ & $32.64 \pm 5.17$ & 5.0 & 0.387 \\
Current smoker (yes) & 14.3 & 27.2 & 0 & 0.412 \\
Current drinker (yes) & 2.3 & 4.5 & 23.8 & 0.469 \\
Physical activity (low) & 43.2 & 40.9 & $26.92 \pm 4.40$ & $<0.001$ \\
Body mass index $\left(\mathrm{kg} / \mathrm{m}^{2}\right)$ & $33.83 \pm 7.30$ & $29.47 \pm 3.66$ & $83.78 \pm 10.27$ & $<0.001$ \\
Waist circumference $(\mathrm{cm})$ & $98.19 \pm 13.81$ & $88.36 \pm 6.58$ & & \\
\hline
\end{tabular}

Value are served as \% for categorical variables or mean \pm SD for continuous variables

Table 5. Characteristics of Participants in Dietary Pattern $3(n=102)$

\begin{tabular}{lllll}
\hline & Dietary pattern 3 & & T3 & p \\
\cline { 2 - 5 } & T1 & T2 & $31.96 \pm 5.53$ & 0.048 \\
\hline Age (years) & $35.92 \pm 6.09$ & $33.97 \pm 6.57$ & 13.8 & 0.160 \\
Current smoker (yes) & 25.9 & 4.2 & 3.3 & 0.457 \\
Current drinker (yes) & 3.1 & 0 & 43.3 & 0.633 \\
Physical activity (low) & 31.3 & 40.0 & $27.91 \pm 5.57$ & $<0.001$ \\
Body mass index $\left(\mathrm{kg} / \mathrm{m}^{2}\right)$ & $34.41 \pm 6.81$ & $31.29 \pm 5.73$ & $85.26 \pm 13.02$ & $<0.001$ \\
Waist circumference $(\mathrm{cm})$ & $97.86 \pm 13.92$ & $92.78 \pm 11.03$ & & \\
\hline
\end{tabular}

Value are served as \% or mean \pm SD for categorical variables or continuous variables

Table 6. Comparison of the Regression Coefficients (95\% Confidence Interval) for the Association of Dietary Patterns and Obesity Parameters ${ }^{\mathrm{a}}$

\begin{tabular}{|c|c|c|c|}
\hline & Dietary Pattern 1 & Dietary Pattern 2 & Dietary Pattern 3 \\
\hline Body mass index & $2.606(0.916-4.296)^{2}$ & $-3.837(-5.533--2.140)^{3}$ & $-3.094(-4.758--1.429)^{3}$ \\
\hline Waist circumference & $4.807(1.813-7.801)^{1}$ & $-7.270(-10.249--4.291)^{3}$ & $-5.603(-8.637--2.569)^{3}$ \\
\hline
\end{tabular}

Table 6 explores the multiple linear regression of anthropometric parameters according to the dietary patterns after adjusting for all the potential confounders. Dietary pattern 1 was positively associated with BMI and WC (all p < 0.05). However, $\mathrm{BMI}$ and $\mathrm{WC}$ were negatively associated with dietary pattern 2 and dietary pattern 3 (all $\mathrm{p}<0.05$ ).

\section{DISCUSSION}

Our results found that dietary pattern 1 was positively associated with obesity parameters including BMI and WC among adults aged 20 years and above with obesity; whereas, dietary pattern 2 and dietary pattern 3 were inversely correlated. Dietary pattern 1 was characterized as high in protein, carbohydrate, saturated fat, sodium, food additives, and calorie intake; therefore, it was equal to the unhealthy dietary pattern. ${ }^{89}$ This study was in line with other studies in a different population that found a linear association of the western pattern with overweight and obesity. ${ }^{15,16}$ Additionally, an unhealthy pattern, which is rich in saturated fat, was positively associated with increased body weight. ${ }^{6} \mathrm{~A}$ dietary pattern characterized by high intake of meat, innards, fat, and processed food was significantly related with increased odds of high waist circumference and high waist-to-hip ratio. ${ }^{7}$
Dietary pattern 2 in this study was characterized as high in antioxidants, fiber and complex carbohydrate, and phytochemicals; therefore, this pattern was comparable with a prudent dietary pattern. The prudent pattern had a protective result on obesity and metabolic diseases. ${ }^{7,17,18}$ The Mediterranean diet, a healthy dietary pattern, is also correlated with reduced the risk of obesity. ${ }^{19}$ This pattern is high in fish, vegetables, legumes, and fruit. ${ }^{19}$ Similar to the healthy diet, dietary pattern 3 was described as rich in antioxidants, fiber, probiotics and prebiotics. ${ }^{7,8,20}$ Whole grain and whole cereal patterns provided many sources of dietary fiber, antioxidants, and prebiotics which had a beneficial effect to body weight and insulin regulation..$^{21,22}$

Generally, dietary patterns emerge more convincing to intercede health issues rather than single food items, in spite of the fact that single food items such as macro or micronutrients had necessary impacts on health outcomes. The mechanisms of dietary pattern effects on obesity status are not fully understood yet. The alteration in intestinal viscosity, absorption of nutrients, production of gut hormones in appetite regulation as well as short-chain fatty acids (SCFA) may influence the association. ${ }^{23}$

The present study supports further information on the relationship between dietary patterns and 
obesity in the adults population. Moreover, we used two parameters of obesity rather than a single parameter only that may strengthen the results. Using only a singular parameter of obesity is known as a weak measure of metabolic diseases. While using some parameters to define obesity have been recommended to affect the association. ${ }^{24,25}$ Nevertheless, our study has a limitation. The crosssectional design may not demonstrate the causal association of diet with obesity, although the trend of the correlation was consistent with the previous study. Therefore, additional researches are required to investigate the correlation between diet and obesity using a longitudinal study.

\section{CONCLUSIONS}

Our study find that dietary patterns play significant role in management of obesity. Dietary pattern 1 was positively associated with BMI and WC among healthy women. While, dietary pattern 2 and dietary pattern 3 were inversely associated with BMI and WC.

\section{ACKNOWLEDGEMENT}

We would like to acknowledge Unit of Research, Community Service and Publication (UP3), Medical Faculty, Universitas Diponegoro for funding this study in 2020.

\section{REFERENCES}

1. Engin A. The definition and prevalence of obesity and metabolic syndrome. Adv Exp Med Biol 2017;960:1-17.

2. Popkin BM, Adair LS, Ng SW. Global nutrition transition and the pandemic of obesity in developing countries. Nutr Rev 2012;70:3-21.

3. Rachmi CN, Li M, Alison Baur L. Overweight and obesity in Indonesia: prevalence and risk factors-a literature review. Public Health 2017; 147:20-9.

4. Zheng W, McLerran DF, Rolland B, Zhang X, Inoue M, Matsuo K, et al. Association between body-mass index and risk of death in more than 1 million Asians. N Engl J Med 2011;364:71929.

5. Kim D, Hou W, Wang F, Arcan C. Factors affecting obesity and waist circumference among US adults. Prev Chronic Dis 2019;16:E02-E.

6. Peng W, Liu Y, Liu Y, Zhao H, Chen H. Major dietary patterns and their relationship to obesity among urbanized adult Tibetan pastoralists. Asia Pac J Clin Nutr 2019;28:507-19.

7. Syauqy A, Hsu C-Y, Rau H-H, Chao J. Association of dietary patterns with components of metabolic syndrome and inflammation among middle-aged and older adults with metabolic syndrome in Taiwan. Nutrients 2018;10:143.

8. Syauqy A, Hsu CY, Rau HH, Chao JC. Association of dietary patterns, anthropometric measurements, and metabolic parameters with C-reactive protein and neutrophil-to-lymphocyte ratio in middle-aged and older adults with metabolic syndrome in Taiwan: a cross-sectional study. Nutr J 2018;17:106.

9. Muga MA, Owili PO, Hsu CY, Rau HH, Chao JC. Association between dietary patterns and cardiovascular risk factors among middle-aged and elderly adults in Taiwan: A populationbased study from 2003 to 2012. PLoS One 2016;11(7): e0157745.

10. Perng W, Fernandez C, Peterson KE, Zhang Z, Cantoral A, Sanchez BN, et al. Dietary patterns exhibit sex-specific associations with adiposity and metabolic risk in a cross-sectional study in urban Mexican adolescents. J Nutr 2017;147:1977-85.

11. Kemenkes RI. Data komposisi pangan Indonesia 2017. [Internet]. Jakarta: Kemenkes RI; 2017 [cited 4 Juni 2018]. Available from: https://www.panganku.org/id-ID/semua_nutrisi.

12. Yulia U, Khusun H, Fahmida U. Dietary patterns of obese and normal-weight women of reproductive age in urban slum areas in Central Jakarta. Brit J Nutr 2016;116:S49-S56.

13. Choo V. WHO reassesses appropriate bodymass index for Asian populations. Lancet 2002;360:235.

14. Verma M, Rajput M, Sahoo SS, Kaur N, Rohilla R. Correlation between the percentage of body fat and surrogate indices of obesity among adult population in rural block of Haryana. J Family Med Prim Care 2016;5:154-9.

15. Naja F, Hwalla N, Itani L, Karam S, Sibai AM, Nasreddine L. A Western dietary pattern is associated with overweight and obesity in a national sample of Lebanese adolescents (13-19 years): a cross-sectional study. $\mathrm{Br} \mathrm{J}$ Nutr 2015;114:1909-19.

16. Esmaillzadeh A, Azadbakht L. Major dietary patterns in relation to general obesity and central adiposity among Iranian women. J Nutr 2008;138:358-63.

17. Castanho GK, Marsola FC, McLellan KC, Nicola M, Moreto F, Burini RC. Consumption of fruit and vegetables associated with the metabolic syndrome and its components in an adult population sample. Cien Saude Colet 2013;18:385-92.

18. Hartley L, Igbinedion E, Holmes J, Flowers N, Thorogood M, Clarke A, et al. Increased consumption of fruit and vegetables for the 
primary prevention of cardiovascular diseases. Cochrane Database Syst Rev 2013(6):CD009874

19. D'Innocenzo S, Biagi C, Lanari M. Obesity and the Mediterranean Diet: A review of evidence of the role and sustainability of the mediterranean diet. Nutrients 2019;11:1306.

20. Karl JP, Saltzman E. The role of whole grains in body weight regulation. Adv Nutr 2012;3:697707.

21. Vetrani C, Costabile G, Luongo D, Naviglio D, Rivellese AA, Riccardi G, et al. Effects of whole-grain cereal foods on plasma short chain fatty acid concentrations in individuals with the metabolic syndrome. Nutrition 2016;32:217-21.

22. Roager HM, Vogt JK, Kristensen M, Hansen LBS, Ibrügger S, Mærkedahl RB, et al. Whole grain-rich diet reduces body weight and systemic low-grade inflammation without inducing major changes of the gut microbiome: a randomised cross-over trial. Gut 2019;68:83-93.

23. Lattimer JM, Haub MD. Effects of dietary fiber and its components on metabolic health. Nutrients 2010;2:1266-89.

24. Noh HM, Oh S, Song HJ, Lee EY, Jeong JY, Ryu $\mathrm{OH}$, et al. Relationships between cognitive function and body composition among community-dwelling older adults: a crosssectional study. BMC Geriatr 2017;17:259.

25. Figley CR, Asem JS, Levenbaum EL, Courtney SM. Effects of body mass index and body fat percent on default mode, executive control, and salience network structure and function. Front Neurosci 2016;10:234. 